\title{
Characterization of Ultra-Long Raman Fibre Lasers
}

\author{
S. A. Babin ${ }^{1}$, V. Karalekas ${ }^{2}$, E. V. Podivilov ${ }^{1}$, \\ V. K. Mezentsev ${ }^{2}$, P. Harper ${ }^{2}$, J. D. Ania-Castañón ${ }^{2,3}$ and S. K. Turitsyn ${ }^{2}$ \\ ${ }^{1}$ Institute of Automation and Electrometry SB RAS, 1 Koptyug ave., Novosibirsk 630090, Russia \\ ${ }^{2}$ Photonics Research Group, Aston University, Birmingham, B4 7ET, UK \\ ${ }^{3}$ Instituto de Óptica "Daza de Valdés", CSIC, Madrid 28006, Spain
}

\begin{abstract}
We present results on characterization of lasers with ultra-long cavity lengths up to $84 \mathrm{~km}$, the longest cavity ever reported. We have analyzed the mode structure, shape and width of the generated spectra, intensity fluctuations depending on length and intra-cavity power. The RF spectra exhibit an ultra-dense cavity mode structure (mode spacing is $1.2 \mathrm{kHz}$ for $84 \mathrm{~km}$ ), in which the width of the mode beating is proportional to the intra-cavity power while the optical spectra broaden with power according to the square-root law acquiring a specific shape with exponential wings. A model based on wave turbulence formalism has been developed to describe the observed effects.
\end{abstract}

Keywords: Fibre laser, Raman, ultra-long, mode structure, spectral broadening, wave turbulence

\section{INTRODUCTION}

Raman fibre lasers (RFLs), which have recently attracted a lot of attention [1-5], utilise the non-linear effect of stimulated Raman scattering (SRS) that manifests itself as a shift of the spectrum of propagating electromagnetic radiation towards longer wavelengths. In contrast to bulk media where the light beam should be focused tightly into the substrate to observe the effect, an optical fibre waveguide geometry naturally provides a much stronger SRS effect due to the much higher intensity-length product. Moreover, the uniquely low absorption of light $(\alpha \sim 0.2 \mathrm{~dB} / \mathrm{km}$ at the wavelength $\lambda \sim 1.55 \mu \mathrm{m}$ corresponding to the transparency window of silica glasses) and small fibre core diameter (5-10 $\mu \mathrm{m})$ result in propagation of high intensity light without significant attenuation, thus providing homogeneous intensity distribution in a kilometer long fibre spans. At the same time, the SRS of the high-intensity wave treated as a pump induces Raman amplification of the red-shifted Stokes wave with coefficient $\sim 1 \mathrm{~dB} /(\mathrm{km} \cdot \mathrm{W})$ that becomes much higher than its attenuation already at relatively low power, $\sim 1 \mathrm{~W}$. As a result, the integral Raman gain over a kilometres-long fiber amounts to several dBs (or tens of percent), being more than enough to achieve lasing if one forms a cavity by adding, e.g. fibre Bragg gratings (FBGs) resonantly reflecting the forward and backward propagating Stokes waves at the fibre ends [1].

In a conventional standard single mode fibre (SMF) with germanium doped silica core, the Raman gain spectrum is rather broad with a maximum at the Stokes wavelength shifted by $\sim 14 \mathrm{THz}$ from the pump wavelength. Depending on the Raman gain medium and the presence of FBGs creating cavities at specific wavelengths, Raman fibre lasers (RFLs) involving single- as well as multiple-order Stokes shifts can be designed to operate at almost any wavelength in the nearIR region (1.1-1.7 micron), pumped by high-power lasers at $\sim 1.06$ micron (e.g. by Yb-doped fibre laser) providing allfibre design. Furthermore, the number of conversion stages from short to long-wavelength edge may be reduced using $\mathrm{P}_{2} \mathrm{O}_{5}$-doped silica fibres, which present a 3-times larger Raman Stokes shift [2], as the gain media. With the availability of high- power CW laser diode pumps, RFLs present excellent candidates for a variety of applications thanks to their unique attributes, which combine wavelength tunability and multi-wavelength operation [3] with design compactness. Besides, Raman fibre lasers offer an opportunity to increase cavity length by orders of magnitude compared to other types of laser.

\footnotetext{
*e-mail: babin@iae.nsk.su
}

Fiber Lasers V: Technology, Systems, and Applications, edited by Jes Broeng, Clifford Headley, Proc. of SPIE Vol. 6873, 68731P, (2008) · 0277-786X/08/\$18 · doi: 10.1117/12.761626 
One of the most important applications appear in optical communications, where RFLs are used both as signal and pump sources in distributed Raman amplified systems (see e.g. [4] and references therein). Recently, a first-order Ramanamplified communication system providing quasi-lossless signal transmission, and based upon the novel concept of ultra-long (75 km) Raman laser architecture has been proposed and implemented (see e.g. [5] and references therein). In such an ultra-long laser configuration, the combined forward- and backward- propagating wave generated at the Stokes wavelength $(\sim 1455 \mathrm{~nm})$ inside the high-Q RFL cavity (formed in the transmission SMF fibre itself) experiences reduced variations along the fibre span. Hence, the generated intra-cavity RFL power can be used as a homogeneous, stable secondary pump to provide a near constant (along the fibre) Raman gain for optical signal transmitted in the vicinities of $1550 \mathrm{~nm}$ within a bandwidth of $36 \mathrm{~nm}$. If the gain is adjusted to be nearly equal to optical loss, a quasi-lossless transmission is easily implemented. Evidently, such a substantial increase of the RFL cavity compared to standard lasers leads to a new interesting class of lasers with potentially different physical mechanisms underlying their operation. In this context the following fundamental questions arise:

1) What is the limit of the cavity length for a fibre laser operation?

2) What are the specific peculiarities and properties of the ultra-long RFLs, in particular, its spectral features such as cavity mode structure, output spectrum shape and corresponding coherent properties of the generated light?

3) What are the temporal properties of the radiation generated in the ultra-long span of the amplifying medium forming RFL cavity?

In this paper we address these questions by characterization of ultra-long RFL including experimental and numerical analysis of the spectral and temporal characteristics with cavity lengths varying from $6.6 \mathrm{~km}$ to $84 \mathrm{~km}$, (incidentally, the current record laser cavity length to our knowledge). We will demonstrate that important characteristics of such ultralong fibre lasers are determined by weak turbulent-like interactions of a huge number (from one million to 100 millions) of longitudinal laser modes.

Wave turbulence is a fundamental nonlinear phenomenon that occurs in a variety of nonlinear dispersive physical systems (see e.g. [6] and references therein). In many wave-bearing systems nonlinear effects are relatively small and the interaction between waves is weak. The weak wave turbulence theory deals with statistical behaviour of a larger number of weakly interacting waves with random phases. There are two primary types of the physical systems with turbulent-like behaviour. The first case, mostly associated with using the term turbulence, corresponds to the so-called fully developed wave turbulence when the scales at which waves are excited (energy is pumped into the system) and disappeared are well separated and the turbulent energy transfer between spectral components in the inertial interval does not depend much on the details of system specifics at the edges of the spectrum [6]. In the second case, the scales at which energy is pumped into the system and that at which waves are disappeared cannot be fully separated and turbulent-like behaviour does not have a well-defined inertial interval. We present an example of optical system - ultra-long fibre lasers - that exhibits weak wave turbulence behaviour of the second type. We demonstrate experimentally and numerically that turbulent-like weak interactions between a huge number of cavity modes are responsible for important practical characteristics of ultra-long Raman fibre lasers such as spectral broadening of the generated radiation.

\section{EXPERIMENT}

The basic design of the studied ultra-long Raman fibre laser is schematically depicted in Fig. 1. Two equal-power depolarized pumps centered at $1365 \mathrm{~nm}$ are launched from both ends of a standard SMF span. Two highly reflective fiber Bragg gratings (FBG) with reflection coefficient $\sim 98 \%$ centered at $1455 \mathrm{~nm}$ and a bandwidth of $\sim 1 \mathrm{~nm}$, are positioned at both ends of the fibre span forming a high-Q cavity that traps the first Stokes wave. When the total power from the primary pump injected into the fibre is above the required threshold for the SRS-induced gain to overcome fibre attenuation, the RFL starts lasing at $1455 \mathrm{~nm}$. In order to study the dependence of RFL properties on cavity length, the following span lengths were studied, $\mathrm{L}=6.6,22,44$ and $84 \mathrm{~km}$. In order to monitor the generated intra-cavity Stokes wave power and its optical and RF spectra, two 99:1 couplers were placed near the FBGs at the right (I) and left (II) ends of the span. The RF spectrum was monitored through the $1 \%$ coupler end using a photodetector and an electrical spectrum analyzer. Intermode beating peaks observed in the RF spectrum enable mode structure analysis (for details see e.g. [7]). To examine the optical spectrum of the generated Stokes wave, an optical spectrum analyzer with resolution of $\sim 0.01 \mathrm{~nm}$ was used. The power of the generated Stokes wave at $1455 \mathrm{~nm}$ was measured by a power meter. The temporal behaviour of the generated RFL power was also monitored using a fast oscilloscope with a resolution of $50 \mathrm{fs}$. 


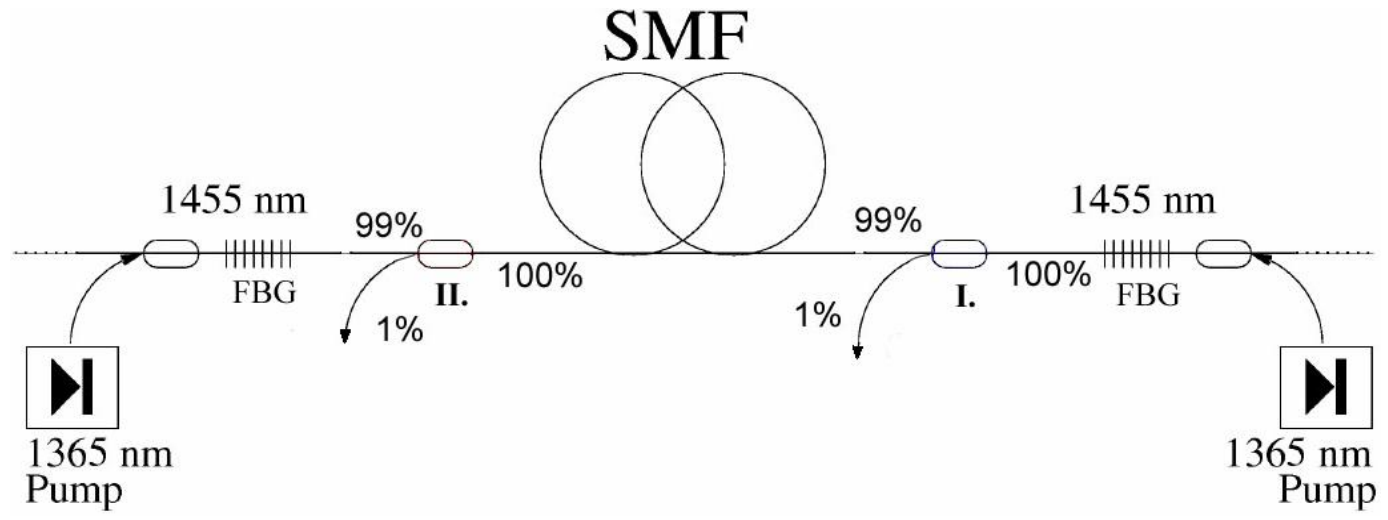

Fig.1. Schematics of the ultra-long Raman fibre laser

Fig. 2a shows the total intra-cavity power of the Stokes wave $(1455 \mathrm{~nm})$; measured via the $1 \%$ port at point II, as a function of the total pump power at $1365 \mathrm{~nm}$. The generated power exhibits typical laser behaviour. At the threshold pump power required for the SRS to overcome the fibre attenuation at $1455 \mathrm{~nm}\left(\alpha_{1455} \approx 0.25 \mathrm{~dB} / \mathrm{km}\right)$ and lumped losses of FBGs, couplers and connections $\left(\delta_{0} \approx 1.4 \mathrm{~dB}\right.$ ), the laser starts generation at $1455 \mathrm{~nm}$. The experimental results are in a good agreement with numerical modelling (solid lines in Fig. 2a) based on computations of the modes dynamics using ordinary differential equations describing four-wave mixing (FWM) interactions. Though the equations are dynamic, the solutions should be treated as stochastic: the FWM nonlinear process engages a huge number of rapidly oscillating terms with different amplitudes and phases making time evolution of any particular cavity mode extremely random. As turbulence implies randomness in temporal behaviour too and the model exhibits stochastic fluctuations involving various time scales. Above the threshold, the generated power grows nearly linearly with increasing pump power. As it is to be expected, we also see that the longer the cavity length becomes, the lower is the generated $1455 \mathrm{~nm}$ power, due to higher cavity losses caused by the attenuation of the pump $\left(\alpha_{1365} \approx 0.31 \mathrm{~dB} / \mathrm{km}\right)$ and the Stokes wave, which lead to a lower average pump power and a higher threshold. Our experimental threshold values are in complete agreement with the simple theoretical RFL model, which was already confirmed by experiments with short fibre spans [8]. For longer spans, distributed losses remain the main factor, and as a result the threshold value remains nearly proportional to the length. Despite this, we would like to point out that even for the longest, record length of $84 \mathrm{~km}$, the threshold pump power is rather moderate, $\sim 0.7 \mathrm{~W}$.

The evolution of the intra-cavity Stokes wave optical spectra is shown in Fig.2b for the case of the $6.6 \mathrm{~km}$ cavity. The spectrum is rather narrow near threshold and broadens significantly as input pump power is increased. Furthermore, it is seen that the broadened spectrum acquires clear exponential tails. This effect was observed for all cavity lengths studied. To trace how the structure of the spectra depends on the boundary condition, we have employed Gaussian apodized FBGs with ripples in the short-wave wing of their reflection profile. As it is seen in Fig. 2b, the generated spectra follow the reflection profile of the FBGs at low powers, but are not influenced by the boundary conditions at higher powers. An interesting feature of the Stokes spectra shown in Fig. $2 \mathrm{~b}$ is the drift towards longer wavelengths with increasing power. By measuring the grating response spectra, we have determined that the drift is caused essentially by the shift of the central wavelength of the cavity FBGs as a result of its thermal expansion. The spectral width of the Stokes wave increases nonlinearly with power, as seen in Fig.3, and does not vary significantly with length for identical powers. The square-root fit appears to describe well dependence on the power for both 3 and $30 \mathrm{~dB}$ spectral widths.

The mechanism of spectral broadening and its dependence on the input power can be understood from the analysis of the interaction between the modes of the generated intra-cavity wave spectrum. In a laser cavity, the spacing between neighboring cavity modes, see e.g. [9], is given by $\Delta=c / 2 L$, where $c$ is the speed of light in the fibre. Therefore, an increase of the cavity length leads to a corresponding reduction of the mode spacing $\Delta$, which manifests itself in the RF spectrum as the distance between the intermode beating peaks, while the width of the peaks is determined by the relative dephasing of the modes (see [7] and references therein). For $L>6 \mathrm{~km}$, the mode spacing is $\Delta<20 \mathrm{kHz}$ and the number of laser modes in a typical optical spectrum width of $\sim 200 \mathrm{GHz}$ (equal. $\sim 2 \mathrm{~nm}$, see Fig.2b), can be estimated as $\mathrm{N}>10^{7}$. Nonlinear interactions via multiple FWM processes during propagation in the long cavity result in the stochastic evolution of the amplitudes and phases of the individual modes [10,11]. 
(a)

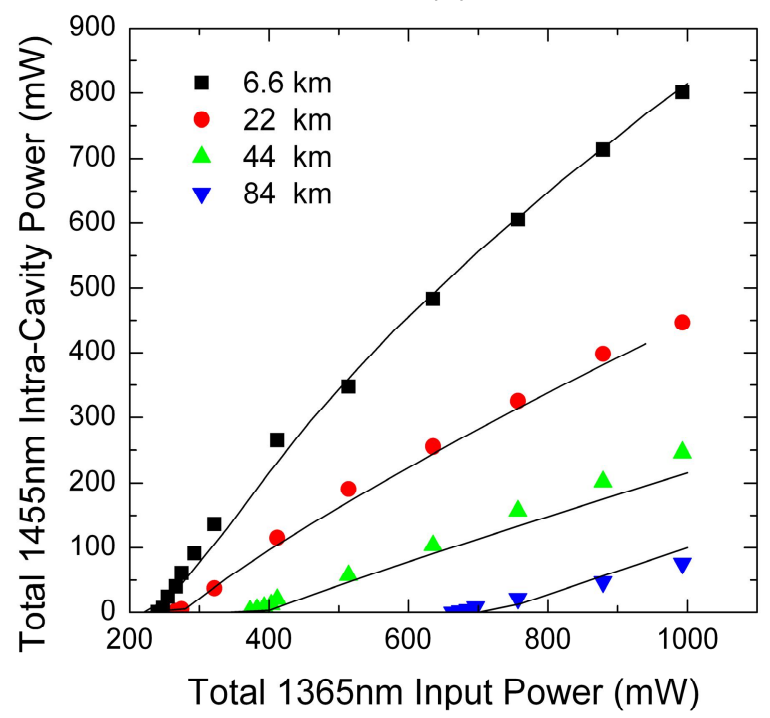

(b)

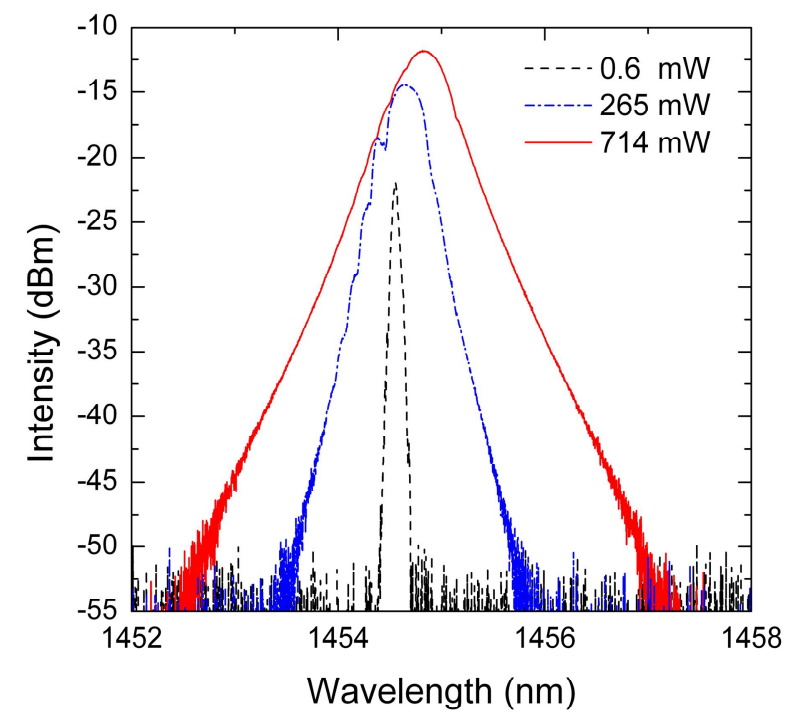

Fig. 2. (a) Total intra-cavity power at $1455 \mathrm{~nm}$ as a function of the total pump power at $1365 \mathrm{~nm}$ : experimental points and numerical simulation (solid curves) . (b) $1455 \mathrm{~nm}$ spectrum measured at point II, I=0.6, 265 and $714 \mathrm{~mW}$ for $\mathrm{L}=6.6$ $\mathrm{km}$.

(a)

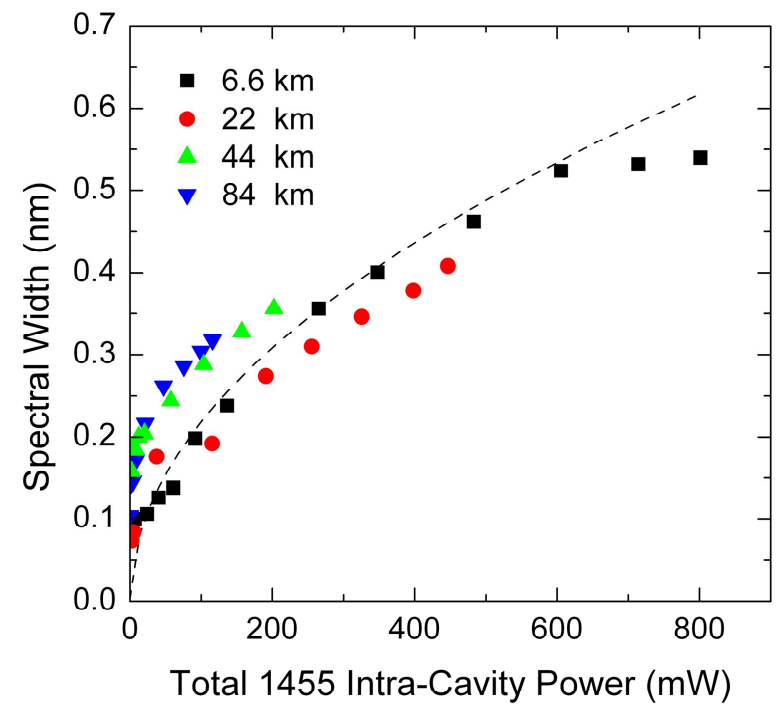

(b)

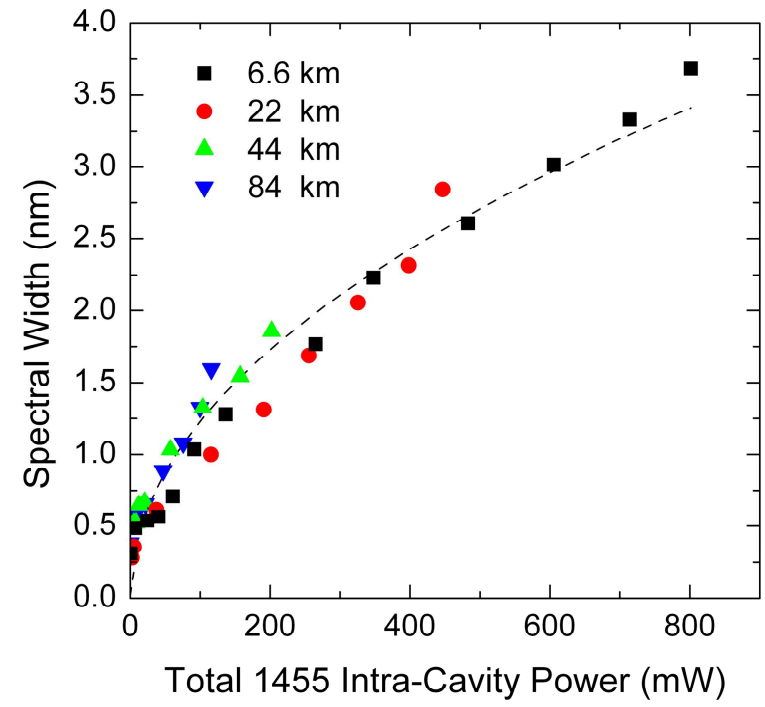

Fig. 3. RFL (1455 nm) spectral spectral widths at $3 \mathrm{~dB}$ (a) and $30 \mathrm{~dB}$ (b) as a function of the intra-cavity (1455 nm) power for different fibre cavity lengths $\mathrm{L}=6.6 ; 22 ; 42$ and $84 \mathrm{~km}$. The dashed lines represent the fit by function $\mathrm{y}=\mathrm{Ax}^{1 / 2}$.

We would like to stress that such a stochastic, turbulent-like behaviour of the modes leads to a rather specific broadening of the RF spectra, proportional to the generated intensity. The width of the intermode beating peaks $D$ appears almost independent of the cavity length and grows linearly with increasing Stokes wave intensity $I$ (see Fig.4a), thus confirming the major role of nonlinear attenuation and stochastic nature of multiple mode interaction in the cavity. At the same time, the measured mode spacing decreases with length, reaching $\Delta=c / 2 L \sim 1.2 \mathrm{kHz}$ for the longest cavity $L=84 \mathrm{~km}$ being tested. So, the power limit (when the mode structure is resolvable, i.e. $D \approx \Delta$ ) is in inverse proportion to the length $I_{\max } \propto L^{-1}$ (Fig. $4 \mathrm{~b}$ ). For the $84 \mathrm{~km}$ cavity it is as low as $\sim 100 \mathrm{~mW}$. Higher powers result in the generation of a "modeless" stochastic spectrum with an exponential-wing envelope. 
(a)

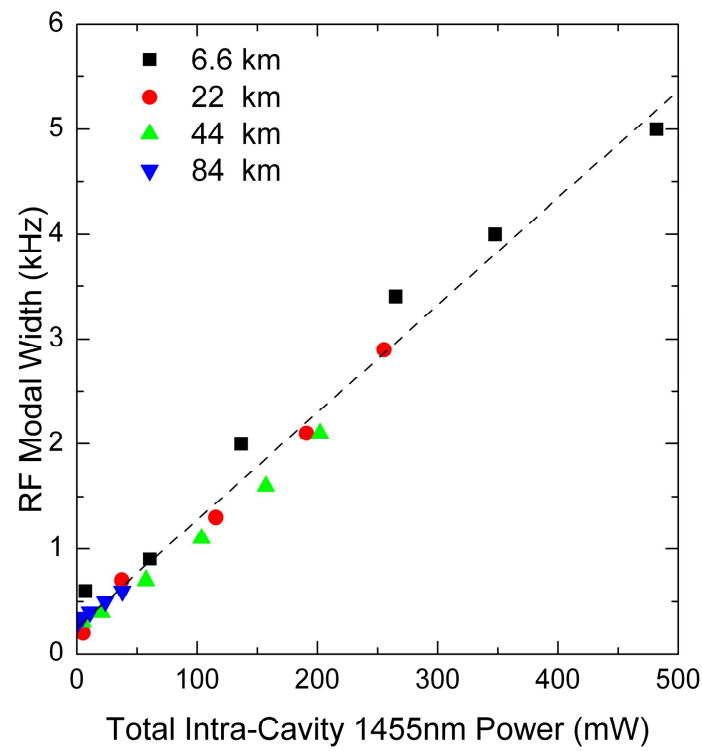

(b)

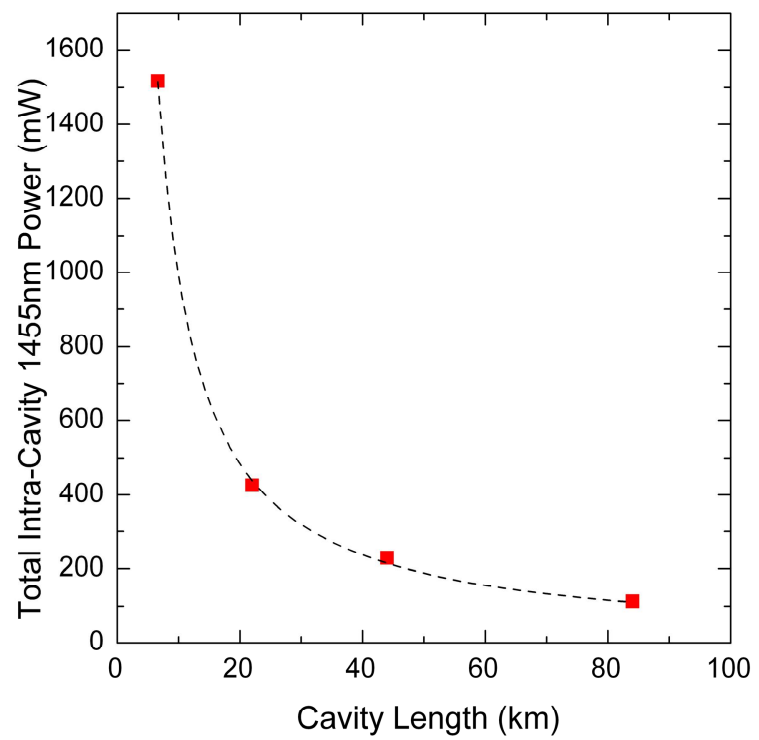

Fig. 4. (a) Spectral width of the RF peaks as a function of the total intra-cavity power at $1455 \mathrm{~nm}$, (b) maximum power with resolved mode structure as a function of the cavity length.

In addition, we have compared the Stokes spectra at high powers just after reflection from the FBG, and after propagation along the fibre, via the right (I) and left (II) couplers (see Fig.5a). It appears that the spectrum reflected from the FBG (inside curve) mimics the FBG spectral reflectivity profile, but after propagation along the whole fibre span it broadens significantly (external curve), besides, the ripples are washed out after propagation through the fibre. At the same time, in the time domain tracked by the fast oscilloscope, we observe, as expected, stochastic behavior with fluctuations in various time scales, Fig.5b. (compare with [12-14]).

(a)

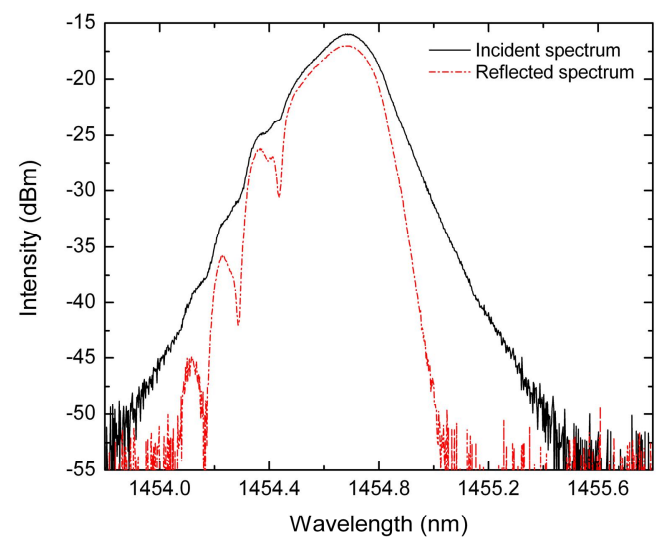

(b)

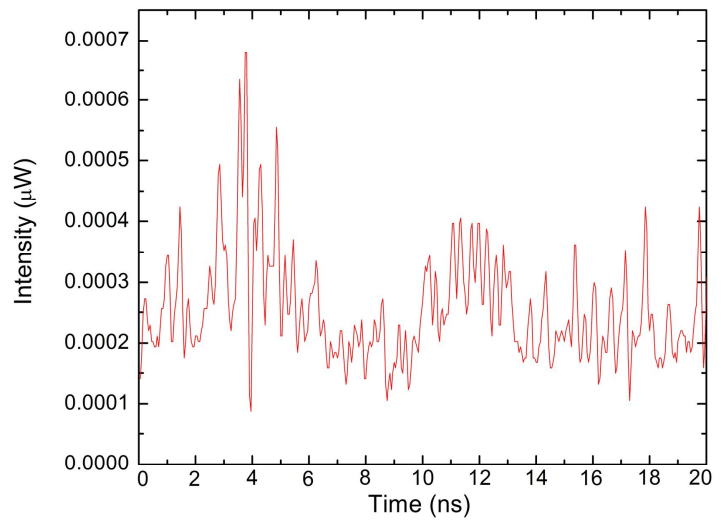

Fig.5. (a) $1455 \mathrm{~nm}$ wave spectrum at I and II ends of the cavity at $400 \mathrm{~mW}$ power, $\mathrm{L}=22 \mathrm{~km}$. (b) $1455 \mathrm{~nm}$ wave temporal behavior at power $\mathrm{I} \sim 100 \mathrm{~mW}, \mathrm{~L}=22 \mathrm{~km}$.

\section{ANALYSIS OF THE RESULTS}

The theoretical analysis of the spectrum formation requires statistical analysis and the use of corresponding statistical techniques instead of the dynamical formalism commonly used in fibre optics, see e.g. [15]. The first attempt in this 
direction has been endeavored in $[10,11]$ for $<1 \mathrm{~km}$ RFL, where the wave kinetic equation for spectral density evolution has been derived. The derivation is based on a technique of averaging and splitting of correlation functions that was developed in the context of the weak turbulence theory applicable to surface, acoustic, spin and other analogue waves [6]. RFL steady state condition, stated as an equality between the integral (over spectrum) gain and losses (lumped plus distributed), allows to obtain an analytical solution under a few natural assumptions. In particular, for longitudinally homogeneous total Stokes wave intensity (that is proved for shorter RFL, see [8]) and typical Gaussian shaped reflection spectrum of the cavity FBGs, the solution for the Stokes wave spectrum generated inside the RFL cavity, takes a hyperbolic secant shape with exponential tales $[10,11]$. Moreover, it has been found that the width of the spectrum grows with increasing power, according to a square-root law [11]. Experimental study of L $0.37 \mathrm{~km}$ long RFL spectra, generated in the region of normal dispersion $(\lambda \sim 1.23 \mu \mathrm{m})$ has demonstrated very good quantitative agreement with the analytical formula both in shape and broadening behavior, observed at Watts power level [11], thus confirming the turbulent nature of the spectral formation.

It is clear that an increase in the length of the laser cavity to $\mathrm{L} \sim 80 \mathrm{~km}$ greatly increases the number of modes $\left(\sim 10^{8}\right)$, thus enhancing the effects of wave turbulence. This is confirmed by the strong broadening of the generated Stokes wave spectra, which remains significant already at $\mathrm{mW}$ level (see Figs.2,3). The width (at $-30 \mathrm{~dB}$ level) reaches $\sim 4 \mathrm{~nm}$ while the power is approaching 1 Watt. The shape of the observed spectrum corresponds qualitatively to the analytical formula of a hyperbolic secant: exponential tales in the logarithmic scale correspond to a linear slope of the wings. Moreover, the dependence of the -3 and $-30 \mathrm{~dB}$ spectral widths as a function of the total intra-cavity power presented in Figure 3 , is nonlinear as predicted by the analytical theory [11]. But the analytical theory is not directly applicable to a qualitative description in case of ultra-long Raman fiber lasers studied here. The main issues not resolved by the simple theory developed in [11] are concerned with the following assumptions: 1) the reflection profile of the FBGs used in the laser cavity is not Gaussian, and have instead characteristic ripples in the reflection spectrum, 2) longitudinal homogeneity is not an adequate assumption for such ultra-long RFL cavity; 3 ) the longer wavelengths, chosen to minimize attenuation $(\lambda \sim 1.455 \mu \mathrm{m})$ correspond to anomalous dispersion instead of normal one.

First, the role of the FBG reflection profile was studied, Fig.5a: the FBG-induced ripples are washed out after the propagation in the fibre. Moreover, we have inserted an additional splitter at intermediate points and have observed that the spectrum acquired its characteristic shape with exponential tails without ripples (outer curve) after $<6 \mathrm{~km}$ propagation in the fibre span and then propagates without significant changes. Therefore, system memory about specific features of FBG reflection profile is lost in the process of nonlinear turbulent evolution of the cavity modes, and exponential tails seem to be a fundamental feature of the process.

For the qualitative analysis of the other features we can use the analytical approach developed earlier [11]. Intra-cavity power $I$ generated at pump power $P$ is derived from the integral equality condition of gain and loss:

$$
2 L \alpha_{I}+\bar{\delta}(I)=2 L g_{R} P_{0} \frac{1-\exp \left(-\alpha_{P} L-g_{R} \frac{\lambda_{I}}{\lambda_{P}} I L\right)}{\alpha_{P} L+g_{R} \frac{\lambda_{I}}{\lambda_{P}} I L} .
$$

The integral gain in RHS is reduced with increasing $I$ because of the pump depletion. Small variations of the intra-cavity power $I(z)$ along the fiber are assumed. Average losses $\bar{\delta}(I)$ in LHS grow with increasing $I$ due to the spectral broadening and corresponding increase of FBG transmission: $\bar{\delta}(I)=\delta_{0}+\delta_{N L}(I)$, where $\delta_{0}$ are effective losses at the central frequency, including lumped losses, $\delta_{N L}(I)$ is an attenuation induced by FWM conversion from the center into the side spectral components. At high power $I$ this conversion defines the width of mode beating in RF spectra: $D=\frac{\delta_{N L}}{\tau_{r t}}=K \gamma c I$, where $\tau_{r t}=2 L / c$ is the round trip time, $\gamma=1.4 \mathrm{~W}^{-1} \mathrm{~km}^{-1}$ is the Kerr nonlinearity coefficient, $\mathrm{c}=2 \cdot 10^{5} \mathrm{~km} / \mathrm{s}$ is the speed of light in the fibre, $\mathrm{K}$ is coefficient depending on the spectrum shape, which can be evaluated as $1 / 5$ in the present conditions, since proportionality coefficient is $K \gamma c=10 \mathrm{kHz} / \mathrm{W}$ (see Fig.5a). 
For Gaussian gratings, $R_{1} R_{2}(\Omega)=\exp \left(-\delta_{0}-\delta_{2} \Omega^{2}\right)$ and the mean-square width of the optical spectrum is proportional to nonlinear losses:

$$
\bar{\Omega}^{2} \approx \frac{\delta_{N L}(I)}{\delta_{2}}=\frac{2 K \gamma L}{\delta_{2}} I
$$

This formula gives an estimation of the width, which appears $\sim 3$ times larger than that observed in the experiment. Note, that we neglected by the backward FWM process: conversion from the side components into the center. Though for normal dispersion domain [11] such neglect is well founded by the stochastic character of FWM interaction, for the domain of anomalous dispersion realized here it is necessary to take into account phase correlations between the waves induced by modulation instability. The distance between correlated components is defined by the modulation instability resonant frequency $\Omega_{M I} \approx \sqrt{\frac{\gamma}{\beta}}$, where $\beta=8.9 \mathrm{~nm}^{-2} \mathrm{~km}^{-1}$ is the dispersion coefficient. This effect evidently limits the resulting spectral width, since this correlation suppresses further broadening and the value $\Omega_{M I} \approx 0.4 \sqrt{I[W]}$ (in [nm] units) could define the spectral width observed in experiments (see Fig.3). This is also confirmed by the observed propagation effects: after reflection from the grating, the spectrum is narrow and mimics the FBG reflection profile with its ripples (Fig. 5.a); then it broadens with propagation, but after several kilometers it takes its final form and propagates without changes.

Since other factors make an analytical approach not applicable to ultra-long RFL, we have performed a numerical simulation of the studied characteristics. The applied simulation model is also based on the standard representation of the Stokes wave as a sum of longitudinal cavity modes, and includes all relevant factors such as the spectral dependence of the FBGs, gain from the pump, distributed cavity loss, and the effect of dispersion on the nonlinear FWM processes with parameters corresponding to experimental conditions: $\delta_{2}=3 \mathrm{~nm}^{-2}, \alpha_{I}=0.25 \mathrm{~dB} / \mathrm{km} \alpha_{P}=0.31 \mathrm{~dB} / \mathrm{km}, g_{R}=0.5 \mathrm{~W} / \mathrm{km}$, $\delta_{0}=1.4 \mathrm{~dB}$ (including all lumped losses).

Deterministic ordinary differential equations describe the modes' dynamics. Though the equations are dynamic, their solution is stochastic: FWM nonlinear process engages a huge number of rapidly oscillating terms with different amplitudes and phases making time evolution of any particular cavity mode extremely stochastic. The results of the simulation were averaged over $\sim 100$ realizations. Simulated spectra have been compared with experimental ones (Figs. 2-5) and show very good qualitative and even quantitative coincidence. The results of the comparison for the spectral width in 22-km long RFL is shown in Fig. 6.

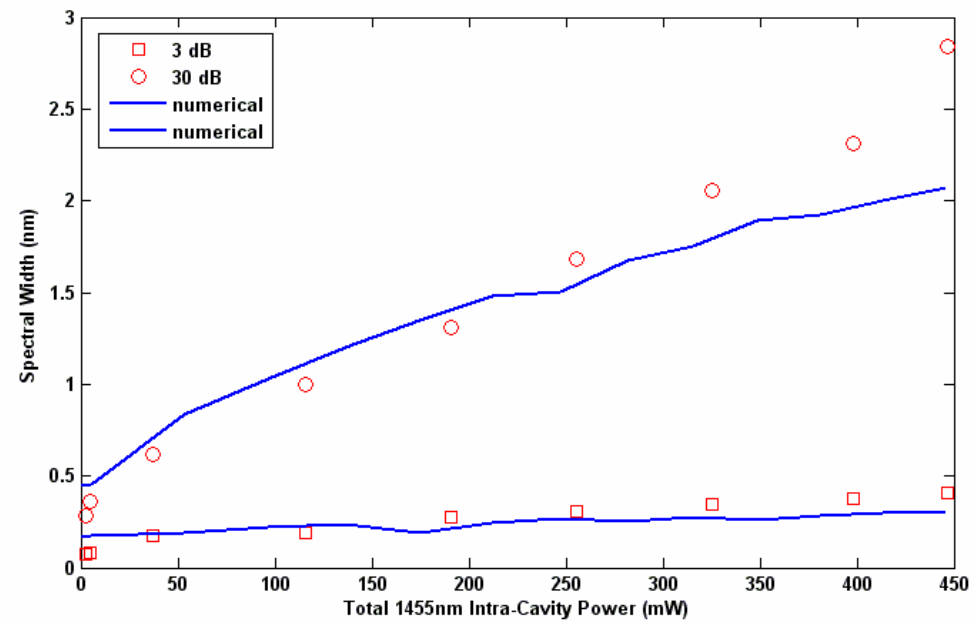

Fig.6. Simulated (lines) and measured (points) $1455 \mathrm{~nm}$ spectral width at $3 \mathrm{~dB}$ (bottom) and $30 \mathrm{~dB}$ (top) level versus 1455 $\mathrm{nm}$ power for $\mathrm{L}=22 \mathrm{~km}$. 
The spectrum broadens with increasing power in a non-linear manner (close to square-root law), while its shape is close to hyperbolic secant with characteristic exponential tails, as the inhomogeneities induced by the FBG ripples are washed out. The only and slight difference concerns the absolute width values, that appears significant for higher powers ( $>300$ $\mathrm{mW}$ at $\mathrm{L}=22 \mathrm{~km}$ ), see Fig.6. The discrepancy remains also larger for longer fibres but taking into account the accuracy of absolute power measurements via coupler (5-10\%) and the increasing role of inhomogeneous distribution of the pump and Stokes waves in the longer fibres and the higher powers, the discrepancy is reasonable. Thus, the numerical model of the turbulence-induced spectral broadening describes the experimental spectra in ultra-long lasers as the analytical model in short ones. As turbulence means a stochasticity in temporal behavior too, it exhibits stochastic fluctuations involving various time scales as reflected in Fig.5b.

\section{CONCLUSION}

In conclusion, the performed experimental and numerical studies have demonstrated a new operation regime of ultralong laser (RFL) - turbulent generation of multitude (up to $\sim 10^{8}$ ) of interacting cavity modes. The observed weak wave turbulence effects are of fundamental interest and have also direct impact on RFL applications in communications, e.g. the performance of quasi-lossless links based on ultra-long Raman lasers [5].

The new class of studied ultra-long lasers is characterized by the following features: by the specific shape (with exponential wings) of the generated optical spectra, which broadens nonlinearly (close to square-root) with increasing intensity and its mode structure, which is resolvable, but only in the limited power interval from the generation threshold up to maximum power which decreases for longer lasers. Above this power value the stochastic mode dephasing leads to fluctuations of the mode frequencies, which become larger than the inter-mode spacing thus leading to the generation of a "quasi-continuous" or "modeless" spectrum. This power limit is $\sim 100 \mathrm{~mW}$ for the $84 \mathrm{~km}$ cavity. Taking into account that more-or-less stable measurable intra-cavity RFL power is $\sim 10 \mathrm{~mW}$, the maximum length of the ultra-long laser with resolvable mode structure may be increased at least by one order of magnitude. In some sense this feature may be used to define the limit length on "ultra-long" fiber lasers: in such laser (at some power level) the light "forgets" about the cavity mode structure during propagation along the fiber, owing to nonlinearity-induced dephasing.

\section{ACKNOLEDGEMENTS}

S.A.B. and E.V.P. acknowledge financial support by the Presidium and the Department of Physical Sciences of the Russian Academy of Sciences and its Siberian Branch (integration project N31). J.D. Ania-Castañón thanks the Engineering and Physical Sciences Research Council for their financial support. The authors also thank A. M. Shalagin for helpful discussions.

\section{REFERENCES}

1 S. G. Grubb, T. Strasser, W. Y. Cheung, W.A. Reed, V. Mizrahi, T. Erdogan, P.J. Lemaire, A.M. Vengsarkar, and D.J. Digiovanni, "High-power, $1.48 \mu \mathrm{m}$ cascaded Raman laser in germanosilicate fibres", in Optical Amplifiers and Their Applications 1995, Proc., Paper SaA4.S7, pp.197-199.

2 E. M. Dianov, M. V. Grekov, I. A. Bufetov, et al. "CW high power $1.24 \mu \mathrm{m}$ and 1,48 $\mu \mathrm{m}$ Raman lasers based on low loss phosphosilicate fibre", Electron. Lett. 33, 1542-1542 (1997).

3 M.D. Mermelstein, C. Headley, J.-C. Bouteiller, P. Steinvurzel, C. Horn, K. Feder, B.J. Eggleton, "Configurable three-wavelength Raman fibre laser for Raman amplification and dynamic gain flattening", IEEE Photon. Techn. Lett. 13, 1286-1288 (2001).

4 C. Headley and G.P. Agrawal, Raman Amplification in Fibre Optical Communication Systems (Academic Press, 2004).

5 J. D. Ania-Castañón, T. J. Ellingham, R. Ibbotson, X. Chen, L. Zhang, and S. K. Turitsyn, ”Ultralong Raman fibre lasers as virtually lossless optical media”, Phys. Rev. Lett. 96, 023902 (2006).

6 V.E.Zakharov, V.S.L'vov, G.Falkovich, "Kolmogorov spectra of turbulence I: Wave turbulence" (SpringerVerlag, 1992).

7 S. A. Babin, V. Karalekas, P. Harper, E. V. Podivilov, V. K. Mezentsev, J. D. Ania-Castañón, S. K. Turitsyn "Experimental demonstration of mode structure in ultra-long Raman fibre lasers", Opt. Lett. 32, 1135-1137 (2007). 
S. A. Babin, D. V. Churkin, and E. V. Podivilov, "Intensity interactions in cascades of a two-stage Raman fibre laser", Opt. Comm. 226, 329-335 (2003).

9 O. Svelto, "Principles of lasers" (Springer; 1 edition, 1999).

10 S. A. Babin, D. V. Churkin, A. E. Ismagulov, S. I. Kablukov and E. V. Podivilov, "Spectral broadening in Raman fibre lasers", Opt. Lett. 31, 3007-3009 (2006).

11 S. A. Babin, D. V. Churkin, A. E. Ismagulov, S. I. Kablukov and E. V. Podivilov, "FWM-induced turbulent spectral broadening in a long Raman fiber laser", JOSA B 24, 1729-1738 (2007).

12 D. Hochheiser, J. V. Moloney, and J. Lega, "Controlling optical turbulence", Phys. Rev. A 55, R4011-R4014 (1997).

13 G. Steinmeyer, A. Schwache, and F. Mitschke, "Quantitative characterization of turbulence in an optical experiment ”, Phys. Rev. E 53, 5399 - 5402 (1996).

14 F. Mitschke, G. Steinmeyer and A. Schwache, " Generation of one-dimensional optical turbulence“, Physica D, 96, 251-258 (1996).

15 G. P. Agrawal, "Nonlinear fiber optics" (Academic press, London, U.K., 1995). 